\title{
Self-gravitating systems of ideal gases in the 1PN approximation
}

\author{
Gilberto M. Kremer ${ }^{1}$ Martín G. Richarte ${ }^{1,2}$ 团 and Karla Weber团 \\ ${ }^{1}$ Departamento de Física, Universidade Federal do Paraná, \\ Caixa Postal 19044, 81531-990 Curitiba, Brazil and \\ ${ }^{2}$ Departamento de Física, Facultad de Ciencias Exactas y Naturales, \\ Universidad de Buenos Aires, Ciudad Universitaria 1428, Pabellón I, Buenos Aires, Argentina
}

\begin{abstract}
We obtain the Maxwell-Jüttner distribution function at first order in the post-Newtonian approximation within the framework of general relativity. Taking into account the aforesaid distribution function, we compute the particle four-flow and energy-momentum tensor. We focus on the search of static solutions for the gravitational potentials with spherical symmetry. In doing so, we obtain the density, pressure and gravitational potential energy profiles in terms of dimensionless radial coordinate by solving the aforesaid equations numerically. In particular, we find the parametric profile for the equation of state $p / \rho$ in terms of the dimensionless radial coordinate. Due to its physical relevance, we also find the galaxy rotation curves using the post-Newtonian approximation. We join two different kinds of static solutions in order to account for the linear regime near the center and the typical flatten behavior at large radii as well.
\end{abstract}

PACS numbers: 04.20.-q, 05.20.Dd, 51.30.+i

\section{INTRODUCTION}

Galaxies are composed of many different kinds of astrophysical objects such as stars, interstellar gas, dust, and dark matter amongst other things. Such system holds together due to gravitational interaction amongst their constituents. In fact, they can be organized in bigger astrophysical structures called clusters or super-clusters [1]. It is generally believed that the amount of dark matter in a cluster is ten times bigger than the total amount of gas and stars [2]. However, the recent analysis based on both optical and radio data in spiral galaxies reveals that the amount of dark matter is even less than previously thought [3]; where the gravitational field associated with spiral galaxies (such as NGC 7793, 1365, 6946 and UGC 6446) is assumed to follow a global disk-like profile rather than spheroidal one [3]. For a detailed analysis of the universal rotational curve of spiral galaxies and its connection with dark matter see [4]. If the physical scale of interest is much bigger than the size of these objects then the latter ones can be considered as pointlike particles interacting among them, the gravity being the most relevant interaction. Hence, one possible route to study this system may be to solve its dynamical equation and find the trajectories of each one of the "particles". Nevertheless, the number of particles involved in these systems is enormously big so the aforesaid approach becomes unsuitable. An alternate view of galaxies is as a system of particles in six dimensional phase space. The galaxies are then instantaneously described in terms of a distribution function $f(x, p)$ over the phase space, where $x$ represents the position vector and $p$ stands for the momentum. In

\footnotetext{
*Electronic address: kremer@fisica.ufpr.br

†Electronic address: martin@df.uba.ar

${ }^{\ddagger}$ Electronic address: karlaw@fisica.ufpr.br
}

this way, galaxies can be described in terms of ensemble of particles along with a distribution function which satisfies a kinetic equation [5]. If one obtains the distribution function associated with the kinetic equation then one can extract the main traits of the system under consideration, for instance, one can be able to reconstruct the average square velocity and several other moments of the distribution function [5].

If the collision between two particles is a very rare event then the collision operator of the Boltzmann equation can be neglected. In the latter case, one can work with the collisionless Boltzmann equation (sometimes this is also known as Vlasov equation) whose solution turns out to be an equilibrium distribution function [5]. As an example, one thinks in a gas with a Boltzmann distribution at equilibrium whose gravity center follows a circular geodesic in Schwarzschild field [6]. Or the case of dark matter halos, dark matter only interacts gravitationally and there are hardly any encounters, so that one can describe the dark matter as a collisionless system and use the Vlasov equation in order to describe its evolution [7]. Besides, a self-consistent rederivation of collisionless Boltzmann equation for self-gravitating gases with post-Newtonian corrections 8] was obtained recently; the case of post-Newtonian polytropes solutions was examined numerically, focusing in the role played by the relativistic correction to the rotation curves [8].

As is well-known the equations of general relativity reduce to those of Newtonian gravity in the limit of slow motions along with weak gravitational fields. Newton's theory of gravity is good enough to describe all the physics in the solar system, but it is incomplete and requires some corrections to properly account for the shift in the perihelion of Mercury 9]. In order to describe physical phenomena like the latter one it becomes essential to include post-Newtonian (PN) corrections to the standard gravitational physics. A straightforward manner to include all the relativistic corrections to New- 
tonian gravity is by means of post-Newtonian formalism. The post-Newtonian method relies on the idea that one perturbs around a Minkowski background. In doing so, one considers that the perturbations of the metric tensor along with those corresponding to the energymomentum tensor are both small fluctuations, which can be expanded in power of $v / c$, being $v$ a typical velocity in a system associated with matter and $c$ is the speed of light [9]. One of the main reasons to examine relativistic corrections in galactic dynamics concerns to the issue of the rotation curves predicted by Newtonian theory. In Newtonian theory, rotation curves typically increase linearly near the origin up to a maximum and then vanish for large radii, while the measured circular velocity curve for the galaxies leads to small value near the center, increases linearly, then exhibits a small cusp and tends to a finite nonzero value at large radii. One can clearly recognize the contribution of the dark matter halo in order to generate the flat circular velocity curve [7]. Indeed, some authors pointed out that the inclusion of post-Newtonian corrections in astrophysical models can really help to reduce the amount of dark matter needed to explain rotation curves which flatten at large radii [10]. Of course, these corrections by their own cannot overcome the whole problem of generating flat rotation curves, however, can reduce the amount of dark matter in relation with the Newtonian models.

For the reasons mentioned above, the study of relativistic corrections within the context of kinetic theory seems to be a very promising route. Bearing this in mind, many authors devoted some efforts to apply kinetic theory plus post-Newtonian method to describe astrophysical models. They explored the kinetic theory of self-gravitating gases with axial symmetry within the framework of post-Newtonian formalism applied to the case of razor thin disks, focusing on axially symmetric galaxy models [1]. In particular, they obtained the rotation curves and mass profiles for the post-Newtonian version of Morgan-Morgan disks and derived the virial theorem in 1PN approximation as well [11]. Continuing with this line of research, Nguyen and Pedraza studied selfgravitating system with polytropic equation of state in the PN approximation of general relativity, the physical motivation is that such equation can be used to describe many astrophysical models such as white dwarfs, neutron stars, galactic halos, and globular clusters amongst others. By solving the Einstein-Vlasov system of equations, they found a family of star clusters with anisotropic in velocity space within $\mathrm{PN}$ scheme. In addition, they analyzed the stability of circular orbits for radial perturbations [12].

Here, we are going to use the relativistic collisionless Boltzmann equation along with the Maxwell-Jüttner distribution function to derive the Maxwell-Jüttner distribution function in the $1 \mathrm{PN}$ approximation. In doing so, we introduce a peculiar velocity associated with the particle velocity in the gas frame, use the Tolman's law and integrate over the peculiar velocity space. We calculate the particle flow at 1PN order while energy-momentum tensor components are derived at different PN orders, for instance, the diagonal temporal part is calculated at $1 \mathrm{PN}$ order but the diagonal spatial components are reported at $2 \mathrm{PN}$ order. In this way, we derive the macroscopic energy momentum tensor from the Maxwell-Jüttner distribution function at 1PN order. Our result coincides with the one obtained from fluids description [9]. We numerically solve the nonlinear equation associated to the gravitational potential fields. Our analysis differs from the one reported for post-Newtonian polytropes in diverse manners [8]. We contrast the Newtonian with the PN profiles of matter density, circular velocity and gravitational potential energy. As was expected, the pressure profile only includes PN corrections. For certain value radius, say $r_{c}$, the gravitational fields become complex and therefore we must match the latter solution with a physical one. We show the procedure to obtain physical gravitational potentials by joining with other solutions at $r_{c}$. In addition, we notice that such method leads to circular velocity profiles that flatten at large radii.

Our paper is outlined as follows. In Sec. II, we give the general derivation of the Maxwell-Jüttner distribution function at $1 \mathrm{PN}$ order. We devote Sec. III to obtain the particle four-flow and energy-momentum tensor within the PN approximation from the Maxwell-Jüttner distribution function at 1PN order. We seek static solution for the gravitational potentials associated with nonlinear Poisson-like equations in Sec. IV. In Sec. V, we solve numerically the aforesaid equations, present the density, pressure and circular velocity profiles. In Sec. VI, we finally reexamine the issue of generating flat rotation curves by gluing two different solutions at certain radius. In Sec. VII the conclusions are stated. Throughout the article we adopt the metric signature $(-,+,+,+)$ and we do not set the speed of light $c$ equal to the unity for practical reasons.

\section{THE MAXWELL-JÜTTNER DISTRIBUTION FUNCTION IN THE 1PN APPROXIMATION}

The kinetic theory of relativistic gases in the presence of gravitational fields is based in the Boltzmann equation (see e.g. [5] and [13]-[16])

$$
p^{\mu} \frac{\partial f}{\partial x^{\mu}}-\Gamma_{\mu \nu}^{i} p^{\mu} p^{\nu} \frac{\partial f}{\partial p^{i}}=\mathcal{C}(f, f) .
$$

Here $f \equiv f(\mathbf{x}, \mathbf{p}, t)$ is the one-particle distribution function in the phase space spanned by the space $\mathbf{x}$ and threemomentum p coordinates, $\Gamma_{\mu \nu}^{i}$ are Christoffel symbols and $\mathcal{C}(f, f)$ is the collision operator of the Boltzmann equation which takes into account the product of the two distribution functions of the colliding particles. In the above expression the mass-shell condition $p^{\mu} p_{\mu}=-m^{2} c^{2}$ - where $m$ is the particle rest mass - was taken into account. 
Equilibrium is characterized by a vanishing collision term $\mathcal{C}(f, f)$ and this implies that the distribution function is the Maxwell-Jüttner distribution function

$$
f=\frac{n}{4 \pi m^{2} c k T K_{2}(\zeta)} \exp \left(\frac{p^{\mu} U_{\mu}}{k T}\right) .
$$

Above $k$ is the Boltzmann constant and $n, T, U^{\mu}$ are the particle number density, the temperature and the four-velocity of the gas, respectively. Furthermore, $\zeta=$ $m c^{2} / k T$ represents the ratio of the rest energy of a particle $m c^{2}$ and the thermal energy of the gas $k T$ and $K_{2}(\zeta)$ denotes the modified Bessel function of second kind. The ultrarelativistic regime of the gas is attained at high temperatures where $\zeta \ll 1$, while the nonrelativistic one occurs at low temperatures where $\zeta \gg 1$. Another expression for the Maxwell-Jüttner distribution function is given in terms of the chemical potential $\mu$ - the Gibbs function per particle - namely,

$$
f=\exp \left(\frac{\mu}{k T}-1+\frac{p^{\mu} U_{\mu}}{k T}\right) .
$$

If we insert the equilibrium distribution function into the left-hand side of the Boltzmann equation (1) we obtain the following restrictions for the temperature and chemical potential fields (see [6])

$$
\begin{gathered}
T \sqrt{-g_{00}}=T_{0}=\text { constant } \\
\mu \sqrt{-g_{00}}=\mu_{0}=\text { constant. }
\end{gathered}
$$

The first equation above is known as Tolman's law [17, 18] while the second one as Klein's law [19] and both were introduced within the framework of phenomenological theories.

From the two expressions for the Maxwell-Jüttner distribution function (2) and (3) we get

$$
\frac{n}{4 \pi m^{2} c k T K_{2}(\zeta)}=\exp \left(\frac{\mu}{k T}-1\right)=\exp \left(\frac{\mu_{0}}{k T_{0}}-1\right),
$$

where the last equality follows from Tolman (4) and Klein (5) laws. Hence we may write the left-hand side of (6) in terms of $n_{0}$ and $T_{0}$, namely,

$$
\frac{n}{4 \pi m^{2} c k T K_{2}(\zeta)}=\frac{n_{0}}{4 \pi m^{2} c k T_{0} K_{2}\left(\zeta_{0}\right)} .
$$

Let us calculate the Maxwell-Jüttner distribution function (2) in the first post-Newtonian approximation (1PN approximation).

First we follow [9] and write the line element

$d s^{2}=-c^{2} d \tau^{2}=g_{00}\left(d x^{0}\right)^{2}+2 g_{0 i} d x^{0} d x^{i}+g_{i j} d x^{i} d x^{j}$,

up to $1 / c^{4}$ order as

$$
\begin{array}{r}
c^{2} d \tau^{2}=\left(1+\frac{2 \phi}{c^{2}}+\frac{2\left(\phi^{2}+\psi\right)}{c^{4}}\right)\left(d x^{0}\right)^{2} \\
-2 \frac{\xi_{i}}{c^{3}} d x^{0} d x^{i}-\left(1-\frac{2 \phi}{c^{2}}\right) \delta_{i j} d x^{i} d x^{j},
\end{array}
$$

thanks to the expressions of the metric tensor components (51) - (55) given in the Appendix A. Next by introducing the four-velocity of the gas particles $u^{\mu}=$ $\left(u^{0}, u^{0} v^{i} / c\right)$ it follows from (9) that up to $1 / c^{4}$ order we have

$$
\begin{aligned}
\frac{u^{0}}{c} & =1+\frac{1}{c^{2}}\left(\frac{v^{2}}{2}-\phi\right) \\
& +\frac{1}{c^{4}}\left(\frac{3 v^{4}}{8}-\frac{5 v^{2} \phi}{2}+\frac{\phi^{2}}{2}-\psi+\xi_{i} v^{i}\right) .
\end{aligned}
$$

The above expression is also valid for the components of the fluid four-velocity $U^{\mu}=\left(U^{0}, U^{0} V^{i} / c\right)$ by replacing $u^{0}$ and $v^{i}$ by $U^{0}$ and $V^{i}$, respectively.

If we introduce the peculiar velocity $\mathbf{W}=\mathbf{v}-\mathbf{V}$ which is the particle velocity in the gas frame - and by considering Tolman's law (4) we get the following relationship

$$
\begin{array}{r}
\frac{g_{\mu \nu} p^{\mu} U^{\nu}}{k T}=-\frac{m c^{2}}{k T_{0}}\left\{1+\frac{W^{2}}{2 c^{2}}+\frac{\phi}{c^{2}}+\frac{1}{c^{4}}\left[\frac{3 W^{4}}{8}\right.\right. \\
-\frac{3 W^{2} \phi}{2}+\frac{(\mathbf{V} \cdot \mathbf{W})^{2}}{2}+\frac{V^{2} W^{2}}{2} \\
\left.\left.+(\mathbf{V} \cdot \mathbf{W}) W^{2}+\frac{\phi^{2}}{2}+\psi\right]\right\} .
\end{array}
$$

Furthermore, up to $1 / c^{2}$ order the modified Bessel function of second kind reads

$$
\frac{1}{K_{2}\left(\zeta_{0}\right)}=\sqrt{\frac{2 m c^{2}}{\pi k T_{0}}} e^{\frac{m c^{2}}{k T_{0}}}\left(1-\frac{15 k T_{0}}{8 m c^{2}}+\ldots\right) .
$$

Hence the Maxwell-Jüttner distribution function (2) in the $1 \mathrm{PN}$ approximation becomes

$$
\begin{array}{r}
f=\frac{n_{0}}{\left(2 \pi m k T_{0}\right)^{\frac{3}{2}}} e^{-\frac{m W^{2}}{2 k T_{0}}-\frac{m \phi}{k T_{0}}}\left\{1-\frac{15 k T_{0}}{8 m c^{2}}\right. \\
-\frac{m}{k T_{0} c^{2}}\left[\frac{3 W^{4}}{8}-\frac{3 W^{2} \phi}{2}+\frac{(\mathbf{V} \cdot \mathbf{W})^{2}}{2}+\frac{V^{2} W^{2}}{2}\right. \\
\left.\left.+(\mathbf{V} \cdot \mathbf{W}) W^{2}+\frac{\phi^{2}}{2}+\psi\right]\right\},
\end{array}
$$

thanks to (10), (11) and (12) and by considering the terms with the factor $1 / c^{2}$ of small order.

\section{MACROSCOPIC DESCRIPTION}

In kinetic theory of relativistic gases the particle fourflow $N^{\mu}$ and the energy-momentum tensor $T^{\mu \nu}$ are given in terms of the distribution function by (see [5])

$$
\begin{gathered}
N^{\mu}=m^{3} c \int u^{\mu} f \frac{\sqrt{-g} d^{3} u}{-u_{0}}, \\
T^{\mu \nu}=m^{4} c \int u^{\mu} u^{\nu} f \frac{\sqrt{-g} d^{3} u}{-u_{0}} .
\end{gathered}
$$


Note that we have written the energy-momentum tensor in terms of the particle four-velocities $u^{\mu}$ instead of the particle four-momentum $p^{\mu}=m u^{\mu}$. Here we follow [5, 13-16] and considered that the element of integration is the invariant $\sqrt{-g} d^{3} p /\left(-p_{0}\right)$ with the covariant component $p_{0}$, which is different from $\sqrt{-g} d^{3} p /\left(p^{0}\right)$ in the 1PN approximation, which was adopted by [8].

In the $1 \mathrm{PN}$ approximation the differential element $d^{3} u$ in terms of the peculiar velocity $\mathbf{W}$ up to the $1 / c^{2}$ order is given by

$$
d^{3} u=\left\{1+\frac{1}{c^{2}}\left[\frac{5\left(V^{2}+2(\mathbf{W} \cdot \mathbf{V})+W^{2}\right)}{2}-3 \phi\right]\right\} d^{3} W .
$$

Furthermore, up to the $1 / c^{2}$ order we can build the relation

$$
\begin{aligned}
& -\left(g_{00} u^{0}+g_{0 i} u^{i}\right)=-g_{00} u^{0}\left(1+\frac{g_{0 i} u^{i}}{g_{00} u^{0}}\right) \\
& \approx u^{0}\left(1+2 \frac{\phi}{c^{2}}\right),
\end{aligned}
$$

thanks to the expressions of the metric tensor given in the Appendix A. Next up to the $1 / c^{2}$ order we have the following relationship

$$
\begin{gathered}
\frac{\sqrt{-g} d^{3} u}{-\left(g_{00} u^{0}+g_{0 i} u^{i}\right)}=\left\{1+\frac{1}{c^{2}}\left[\frac{5\left(V^{2}+2(\mathbf{W} \cdot \mathbf{V})+W^{2}\right)}{2}\right.\right. \\
-7 \phi]\} \frac{d^{3} W}{u^{0}}
\end{gathered}
$$

Once we know the distribution function in the $1 \mathrm{PN}$ approximation we can calculate the particle four-flow and the energy-momentum tensor in this approximation. The particle four-flow $N^{\mu}=\sum_{n} \stackrel{n}{N}^{\mu}$ and the energymomentum tensor $T^{\mu \nu}=\sum_{n} T^{n}{ }^{\mu \nu}$ are split in different orders of the ratio $(\bar{v} / c)^{n}($ see [9] ) where $\bar{v}$ can be identified with the thermal velocity of a particle $\bar{v}=\sqrt{k T_{0} / m}$.

First from (14) together with (13) and (18) we get through integration of the resulting equation that the time component of the particle four-flow becomes

$$
\begin{aligned}
& N^{0}=n U^{0}=\stackrel{0}{N^{0}}+\stackrel{2}{N^{0}}=n_{0} c e^{-\frac{m \phi}{k T_{0}}} \\
& \quad \times\left\{1+\frac{1}{c^{2}}\left[\frac{V^{2}}{2}-\frac{5 \phi}{2}-\frac{m}{k T_{0}}\left(\frac{\phi^{2}}{2}+\psi\right)\right]\right\} .
\end{aligned}
$$

From the above equation and from the expression for $U^{0}$ it is easy to obtain that the particle number density is given by

$$
n=n_{0} e^{-\frac{m \phi}{k T_{0}}}\left\{1-\frac{1}{c^{2}}\left[\frac{3 \phi}{2}+\frac{m}{k T_{0}}\left(\frac{\phi^{2}}{2}+\psi\right)\right]\right\} .
$$

Hence we can write (19) as

$$
\stackrel{0}{N^{0}}+\stackrel{2}{N^{0}}=n c\left[1+\frac{1}{c^{2}}\left(\frac{V^{2}}{2}-\phi\right)\right]
$$

Following the same methodology the space components of the particle number density read

$\stackrel{1}{N^{i}}+\stackrel{3}{N^{i}}=\left(\stackrel{0}{N^{0}}+\stackrel{2}{N^{0}}\right) \frac{V^{i}}{c}=n V^{i}\left[1+\frac{1}{c^{2}}\left(\frac{V^{2}}{2}-\phi\right)\right]$.

The components of the energy-momentum tensor (15) are obtained in the same manner, yielding

$$
\begin{aligned}
\stackrel{0}{T}^{00}+\stackrel{2}{T}^{00} & =\varepsilon\left[1+\frac{1}{c^{2}}\left(V^{2}-2 \phi\right)\right], \\
\stackrel{1}{T}^{0 i}+\stackrel{3}{T}^{0 i} & =\frac{V^{i}}{c}\left[\varepsilon+p+\frac{\varepsilon}{c^{2}}\left(V^{2}-2 \phi\right)\right], \\
\stackrel{2}{T}^{i j}+\stackrel{4}{T}^{i j} & =p\left[1+\frac{2 \phi}{c^{2}}\right] \delta^{i j} \\
& +\left[\varepsilon+p+\frac{\varepsilon}{c^{2}}\left(V^{2}-2 \phi\right)\right] \frac{V^{i} V^{j}}{c^{2}} .
\end{aligned}
$$

In order to derive the above expressions (23) - (25), we have used several nontrivial identities related with the integration of different moments for a Gaussian distribution, see Appendix B for further details. Here the energy density $\varepsilon=\rho c^{2}$ - where $\rho$ is the mass density - and the pressure $p$ are given by

$$
\varepsilon=\rho c^{2}=m n c^{2}\left(1+\frac{3 k T}{2 m c^{2}}\right), \quad p=n k T .
$$

The expressions (23) - (25) are the same as those which are obtained from the representation of the energymomentum tensor

$$
T^{\mu \nu}=p g^{\mu \nu}+(\varepsilon+p) \frac{U^{\mu} U^{\nu}}{c^{2}},
$$

by considering the metric tensor and the four-velocity in the PN approach up to the fourth order (cf. [9]).

\section{THE SEARCH FOR STATIC SOLUTIONS}

In this section we search for static solutions of Einstein's field equations, which in the 1PN approximation reduces to the following equations for the gravitational potentials (see e.g. [9])

$$
\nabla^{2} \phi=\frac{4 \pi G}{c^{2}} \stackrel{0}{T^{00}}, \quad \nabla^{2} \psi=4 \pi G\left(\stackrel{2}{T^{00}}+\stackrel{2}{T^{i i}}\right) .
$$

We follow [8] and integrate the components of the energy-momentum tensor (15) in the range $\left[0, v_{e}\right]$, where $v_{e}$ is the escape velocity. The escape velocity is calculated from the expression for the energy in the $1 \mathrm{PN}$ approximation [8]

$$
E=m\left(\frac{v^{2}}{2}+\phi+\frac{3 v^{4}}{8 c^{2}}-\frac{3 v^{2} \phi}{2 c^{2}}+\frac{\phi^{2}}{2 c^{2}}+\frac{\psi}{c^{2}}\right),
$$

by considering that a gas particle attains its maximum value at $E=0$, so that the gas particle is unable to leave 
the distribution of matter. According to [8] to be consistent with the 1PN approximation the escape velocity must given by $v_{e}=\sqrt{-2 \phi}$. Now by considering a vanishing gas velocity $\mathbf{V}=\mathbf{0}$ we can perform the integrations and get the following results for the components of the energy-momentum tensor in the different orders of the ratio $(\bar{v} / c)^{n}$ :

$$
\begin{array}{r}
\stackrel{0}{0}^{00}=-\rho_{0} c^{2}\left[2 \sqrt{-\frac{\phi_{*}}{\pi}}-e^{-\phi_{*}} \operatorname{erf}\left(\sqrt{-\phi_{*}}\right)\right], \\
\stackrel{2}{T}^{00}=-\frac{\rho_{0} k T_{0}}{m}\left[\left(3-\frac{23}{2} \phi_{*}-10 \phi_{*}^{2}-2 \psi_{*}\right) \sqrt{-\frac{\phi_{*}}{\pi}}\right. \\
\left.-\left(\frac{3}{2}-\frac{7}{2} \phi_{*}-\frac{1}{2} \phi_{*}^{2}-\psi_{*}\right) e^{-\phi_{*}} \operatorname{erf}\left(\sqrt{-\phi_{*}}\right)\right], \\
T^{i i}=-\frac{\rho_{0} k T_{0}}{m}\left[\left(6-4 \phi_{*}\right) \sqrt{-\frac{\phi_{*}}{\pi}}\right. \\
\left.-3 e^{-\phi_{*}} \operatorname{erf}\left(\sqrt{-\phi_{*}}\right)\right] .
\end{array}
$$

In the above equations we have introduced the dimensionless quantities

$$
\phi_{*}=\frac{m}{k T_{0}} \phi, \quad \psi_{*}=\left(\frac{m}{k T_{0}}\right)^{2} \psi .
$$

Furthermore, $\operatorname{erf}\left(\sqrt{-\phi_{*}}\right)$ is the error function and $\stackrel{2}{T}^{i i}$ refers to the trace of $\stackrel{2}{T}^{i j}$. We identify $\stackrel{0}{T}^{00}$ with the energy density $\rho_{0} c^{2}$, while $\stackrel{2}{T^{00}}$ and $\stackrel{2}{T}^{i i}$ with the pressure $\rho_{0} k T_{0} / m$.

We insert (30) - (32) into (28) and get the following coupled system of equations for the gravitational potentials

$$
\begin{array}{r}
\nabla^{2} \phi_{*}=-k_{J}^{2}\left[2 \sqrt{-\frac{\phi_{*}}{\pi}}-e^{-\phi_{*}} \operatorname{erf}\left(\sqrt{-\phi_{*}}\right)\right], \\
\nabla^{2} \psi_{*}=-k_{J}^{2}\left[\left(9-\frac{31}{2} \phi_{*}-10 \phi_{*}^{2}-2 \psi_{*}\right) \sqrt{-\frac{\phi_{*}}{\pi}}\right. \\
\left.-\left(\frac{9}{2}-\frac{7}{2} \phi_{*}-\frac{1}{2} \phi_{*}^{2}-\psi_{*}\right) e^{-\phi_{*}} \operatorname{erf}\left(\sqrt{-\phi_{*}}\right)\right],
\end{array}
$$

where $k_{J}=\sqrt{4 \pi G \rho_{0}} / \bar{v}$ can be identified as the Jeans wavenumber.

The gravitational potentials are only functions of the radial coordinate $r$ so that system of equations (34) and (35) in spherical coordinates can written as

$$
\begin{array}{r}
\frac{1}{\widetilde{r}^{2}} \frac{d}{d \widetilde{r}}\left(\widetilde{r}^{2} \frac{d \widetilde{\phi}}{d \widetilde{r}}\right)=\left[2 \sqrt{\frac{\widetilde{\phi}}{\pi}}-e^{\widetilde{\phi}} \operatorname{erf}(\sqrt{\widetilde{\phi}})\right], \\
\frac{1}{\widetilde{r}^{2}} \frac{d}{d \widetilde{r}}\left(\widetilde{r}^{2} \frac{d \widetilde{\psi}}{d \widetilde{r}}\right)=\left[\left(9+\frac{31}{2} \widetilde{\phi}-10 \widetilde{\phi}^{2}+2 \widetilde{\psi}\right) \sqrt{\frac{\tilde{\phi}}{\pi}}\right. \\
\left.-\left(\frac{9}{2}+\frac{7}{2} \widetilde{\phi}-\frac{1}{2} \widetilde{\phi}^{2}+\widetilde{\psi}\right) e^{\widetilde{\phi}} \operatorname{erf}(\sqrt{\widetilde{\phi}})\right] .
\end{array}
$$

Here the new dimensionless quantities are $\widetilde{r}=r k_{J}, \widetilde{\phi}=$ $-\phi_{*}$ and $\widetilde{\psi}=-\psi_{*}$.

The system of equations (36) and (37) can be solved numerically by specifying appropriate boundary conditions. Here we follow [8] and assume that at the center of the configuration the boundary conditions for the gravitational potentials are:

$$
\widetilde{\phi}(0)=\widetilde{\psi}(0)=1,\left.\quad \frac{d \widetilde{\phi}}{d \widetilde{r}}\right|_{\widetilde{r}=0}=\left.\frac{d \widetilde{\psi}}{d \widetilde{r}}\right|_{\widetilde{r}=0}=0
$$

\section{ANALYSIS OF SOME FIELDS IN THE NEWTONIAN AND 1PN APPROXIMATIONS}

As in the work 8] we shall analyze in this section the profiles of the mass density, velocity of test particles in circular motion and potential energy as functions of the radial distance.

The mass density can be read from (30) and 31 and written as a sum of a Newtonian $\widetilde{\rho}_{\mathrm{N}}$ and a postNewtonian $\widetilde{\rho}_{\mathrm{PN}}$ contribution as

$$
\begin{aligned}
& \widetilde{\rho}=\frac{\rho}{\rho_{0}}=\widetilde{\rho}_{\mathrm{N}}+\widetilde{\rho}_{\mathrm{PN}}, \quad \text { where } \\
& \widetilde{\rho}_{\mathrm{N}}=e^{\widetilde{\phi}} \operatorname{erf}(\sqrt{\widetilde{\phi}})-2 \sqrt{\frac{\widetilde{\phi}}{\pi}} \\
& \widetilde{\rho}_{\mathrm{PN}}=\frac{1}{\zeta_{0}}\left[\left(\frac{3}{2}+\frac{7}{2} \widetilde{\phi}-\frac{1}{2} \widetilde{\phi}^{2}+\widetilde{\psi}\right) e^{\widetilde{\phi}} \operatorname{erf}(\sqrt{\widetilde{\phi}})\right. \\
& \left.-\left(3+\frac{23}{2} \widetilde{\phi}-10 \widetilde{\phi}^{2}+2 \widetilde{\psi}\right) \sqrt{\frac{\tilde{\phi}}{\pi}}\right] .
\end{aligned}
$$

In Fig. 11 it is plotted the dimensionless mass density $\widetilde{\rho}$ as function of the dimensionless radial distance $\widetilde{r}$ for the Newtonian and post-Newtonian approximations. The post-Newtonian approximation is a function of $\zeta_{0}=m c^{2} / k T_{0}$, the ratio of the rest energy of the gas particles and the thermal energy of the gas. One can infer from this figure that the contributions to the mass density becomes larger at the configuration center by decreasing the value $\zeta_{0}$, i.e., by increasing the temperature of the gas $T_{0}$. All mass densities tend to zero for large values of the radial distance $\widetilde{r}$. Opposed to the case where the distribution function is characterized by a polytropic function of the energy 8], here none of the mass densities in the $1 \mathrm{PN}$ approximation become negative. However, the solutions for the potentials for values larger than $\widetilde{r} \approx 3.6$ become complex.

The determination of post-Newtonian corrections to the rotation curves is based on the equation for the acceleration of a free falling particle [8, 9], which for static fields reads

$$
\frac{d \mathbf{v}}{d t}=-\nabla\left(\phi+\frac{2 \phi^{2}+\psi}{c^{2}}\right)+\frac{4 \mathbf{v}}{c^{2}} \mathbf{v} \cdot \nabla \phi-\frac{v^{2}}{c^{2}} \nabla \phi
$$




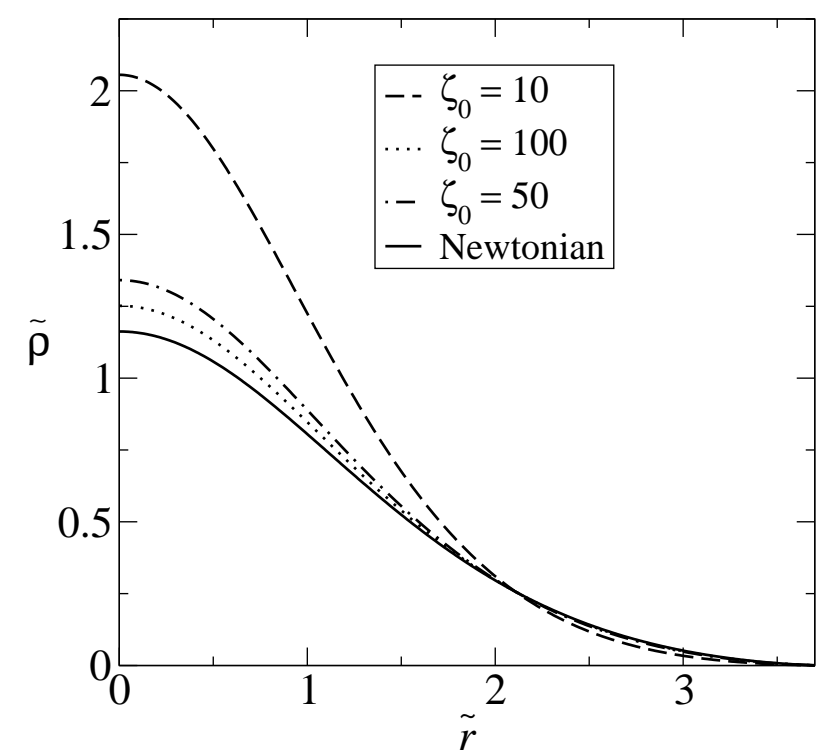

FIG. 1: Mass density $\widetilde{\rho}$ as function of the radial distance $\widetilde{r}$. Newtonian: straight line; Post-Newtonian: dotted line $\zeta_{0}=100$, dash-dotted line $\zeta_{0}=50$, dashed line $\zeta_{0}=10$.

The radial component of the above equation in spherical coordinates $(r, \theta, \varphi)$ for circular orbits of particles in the equatorial plane where $\dot{r}=0, \dot{\theta}=0$ and $\theta=\pi / 2$ reduces to

$$
r \dot{\varphi}^{2}\left(1-\frac{r}{c^{2}} \frac{\partial \phi}{\partial r}\right)=\frac{\partial \phi}{\partial r}+\frac{4 \phi}{c^{2}} \frac{\partial \phi}{\partial r}+\frac{1}{c^{2}} \frac{\partial \psi}{\partial r} .
$$

The expression for the circular velocity $v_{\varphi}=r \dot{\varphi}$ in terms of the gravitational potentials follows from the above equation by considering terms up to the $1 / c^{2}$ order, yielding

$$
v_{\varphi}=\sqrt{r \frac{\partial \phi}{\partial r}\left(1+\frac{4 \phi}{c^{2}}+\frac{r}{c^{2}} \frac{\partial \phi}{\partial r}\right)+\frac{r}{c^{2}} \frac{\partial \psi}{\partial r}} .
$$

Note that the Newtonian circular velocity is given by $v_{\varphi}=\sqrt{r \partial \phi / \partial r}$.

Now in terms of the tilde variables the circular velocity becomes

$$
\widetilde{v}_{\varphi}=\sqrt{\widetilde{r} \frac{\partial \widetilde{\phi}}{\partial \widetilde{r}}\left(\frac{4 \widetilde{\phi}}{\zeta_{0}}+\frac{\widetilde{r}}{\zeta_{0}} \frac{\partial \widetilde{\phi}}{\partial \widetilde{r}}-1\right)-\frac{\widetilde{r}}{\zeta_{0}} \frac{\partial \widetilde{\psi}}{\partial \widetilde{r}}}
$$

where $\widetilde{v}_{\varphi}=v_{\varphi} \sqrt{m / k T_{0}}$ denotes the dimensionless circular velocity.

The dimensionless circular velocity $\widetilde{v}_{\varphi}$ as a function of the dimensionless radial coordinate $\widetilde{r}$ is plotted in Fig. 2. The profiles of the circular velocity have the same behaviors for the Newtonian and post-Newtonian approximations, but for the post-Newtonian approximations the circular velocities have large values. Furthermore, as the mass density, the large values of the circular velocity are attained for smaller values of the parameter $\zeta_{0}$, which correspond to large values of the temperature $T_{0}$. The behavior of the circular velocity for ideal gases differs from the one where the distribution function is characterized by a polytropic function of the energy [8], since in the latter case the values of the circular velocity in the $1 \mathrm{PN}$ approximation are smaller than the corresponding Newtonian approximation. Here the large values of the circular velocity in the $1 \mathrm{PN}$ approximation are due to the fact that the increase of the temperature of the gas, increases the thermal velocity of the particles of the gas $\sqrt{k T_{n} / m}$.

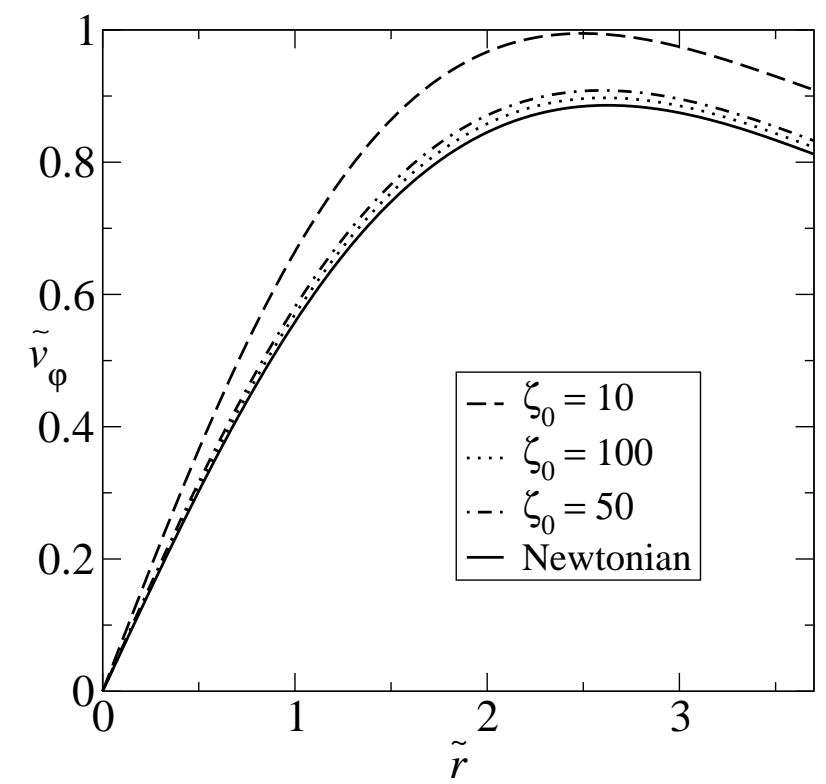

FIG. 2: Circular velocity $\widetilde{v}_{\varphi}$ as function of the radial distance $\widetilde{r}$. Newtonian: straight line; Post-Newtonian: dotted line $\zeta_{0}=100$, dash-dotted line $\zeta_{0}=50$, dashed line $\zeta_{0}=10$.

Another field that can be analyzed is the gravitational potential energy of a gas particle, which can be obtained from the expression for the particle energy (29) by taking $\mathbf{v}=\mathbf{0}$. In terms of dimensionless quantities the Newtonian $\widetilde{U}_{\mathrm{N}}$ and post-Newtonian $\widetilde{U}_{\mathrm{PN}}$ gravitational potential energy read

$$
\begin{array}{r}
\widetilde{U}=\frac{U}{k T_{0}}=\widetilde{U}_{\mathrm{N}}+\widetilde{U}_{\mathrm{PN}}, \quad \text { where } \\
\widetilde{U}_{\mathrm{N}}=-\widetilde{\phi}, \quad \widetilde{U}_{\mathrm{PN}}=\frac{1}{\zeta_{0}}\left(\frac{\widetilde{\phi}^{2}}{2}-\widetilde{\psi}\right) .
\end{array}
$$

In Fig. 3 it is shown the graphic representation of the dimensionless gravitational potential energy $\widetilde{U}$ as function of the dimensionless radial distance $\widetilde{r}$. We can infer from this figure that the Newtonian gravitational potential energy is always negative, while the post-Newtonian 
gravitational potential energies change their sign for large values of the radial distance from the configuration center. The temperature of the gas in the post-Newtonian term $\widetilde{\phi}^{2} / 2 \zeta_{0}$ determines the sign change of the gravitational potential energy. The gravitational potential energy exhibits the same behavior as the one which takes into account a polytropic function of the energy for the

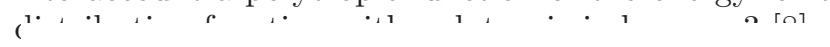

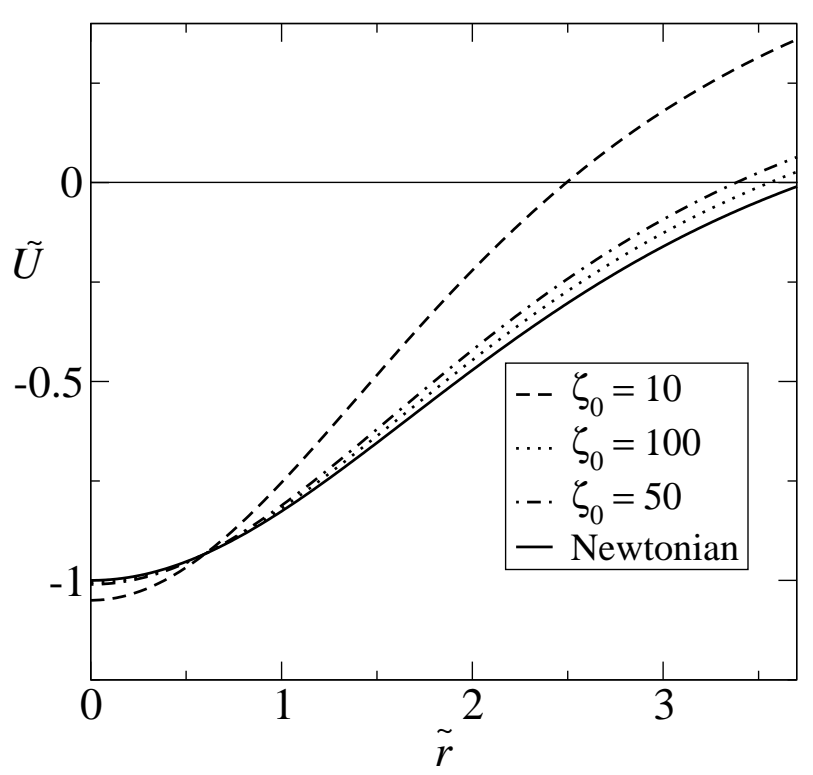

FIG. 3: Gravitational potential energy $\widetilde{U}$ as function of the radial distance $\widetilde{r}$. Newtonian: straight line; Post-Newtonian: dotted line $\zeta_{0}=100$, dash-dotted line $\zeta_{0}=50$, dashed line $\zeta_{0}=10$.

From the energy-momentum tensor given in (32) one can obtain the expression for the pressure of the gas through $p=\stackrel{2}{T}^{i i} / 3$. In terms of dimensionless quantities we have

$$
\widetilde{p}=\frac{m p}{k \rho_{0} T_{0}}=e^{\widetilde{\phi}} \operatorname{erf}(\sqrt{\widetilde{\phi}})-\left(2+\frac{4}{3} \widetilde{\phi}\right) \sqrt{\frac{\tilde{\phi}}{\pi}} .
$$

The dimensionless pressure $\tilde{p}$ as function of the dimensionless radial coordinate $\tilde{r}$ is plotted in Fig. 4. The behavior of the pressure is the same as the one for the mass density, its maximum value occurs at the configuration center and it tends to zero for large values of the radial distance. Note that in the $1 \mathrm{PN}$ approximation the pressure has no contribution which depends on the factor $1 / \zeta_{0}$, since these contributions will appear in the order of $\stackrel{4}{T}^{i i}$. Fig. 5 shows the behavior of the ratio $p / \rho$ in terms of dimensionless radial coordinate. For small radii, $p / \rho$ goes to a constant value whereas tends to zero at large radii.

As a final remark we can ask about the influence of the boundary conditions in the behavior of the analyzed

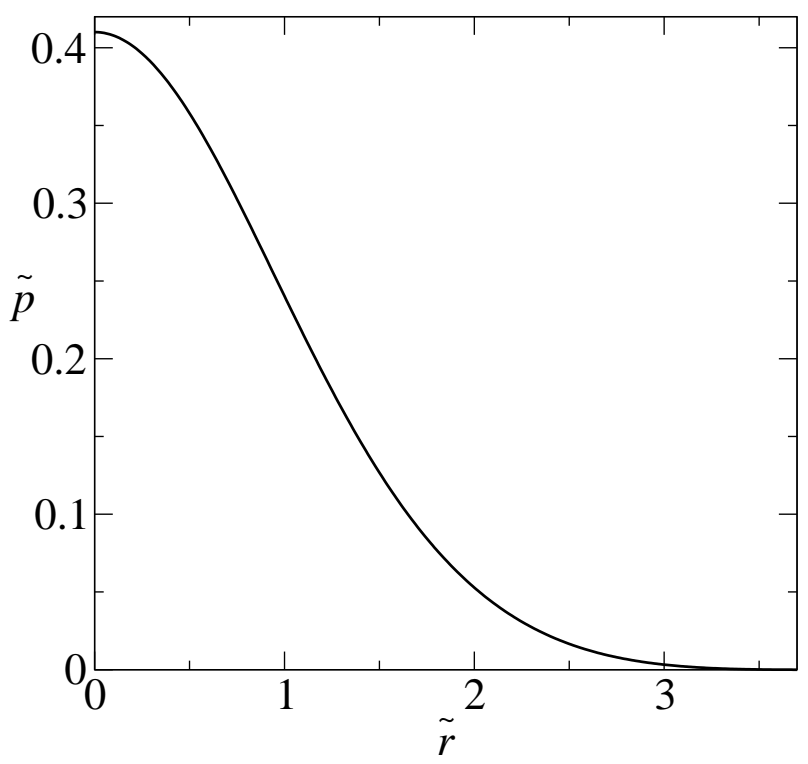

FIG. 4: Pressure $\widetilde{p}$ as function of the radial distance $\widetilde{r}$.

fields. It was found that the boundary condition that has more influence on the solutions refers to the Newtonian gravitational potential $\widetilde{\phi}$ at $\widetilde{r}=0$. However by considering values of $\widetilde{\phi}(0)$ in the range $[0.5,3]$ the behavior of the curves does not change, only the absolute values of the fields become smaller or larger than the ones obtained for $\widetilde{I}(n) \quad 1$

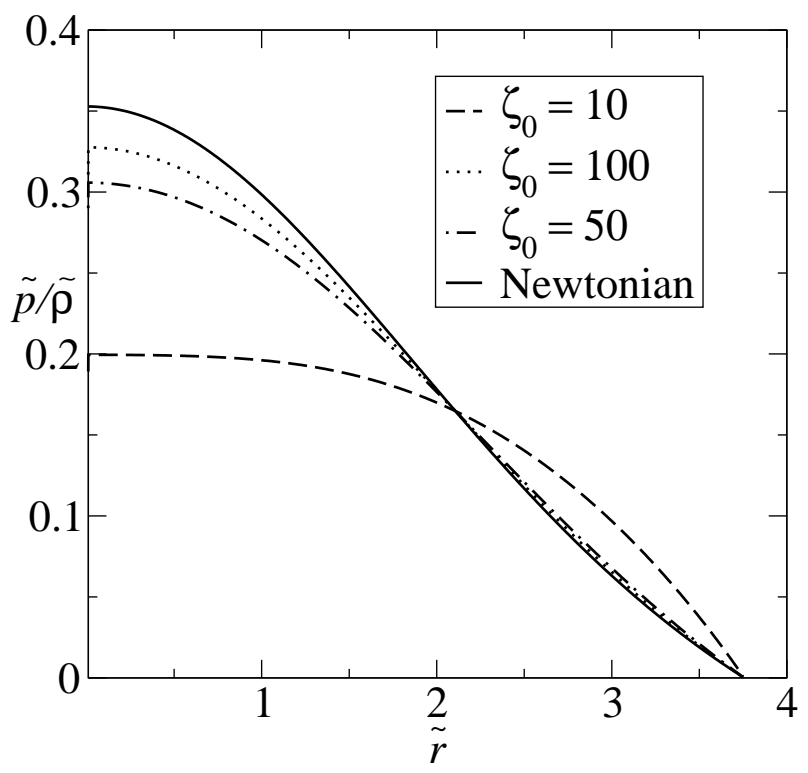

FIG. 5: Pressure-density ratio as function of the radial distance $\widetilde{r}$ for different values of $\zeta_{0}$ 


\section{CIRCULAR ROTATION CURVE}

As we mentioned in Sec. I, one of the most challenging puzzles of current astrophysics is to account for the gravitational mass associated with large structures such as galaxies and cluster or super-cluster of galaxies. It is well-known that the rotation curves of galaxies do not follow a Newtonian profile, which indicates that Newtonian gravity fails to predict the mass distribution of such objects. One way to explain such mismatch is by introducing an unknown component with zero pressure dubbed dark matter. This component cannot be observed or measured directly but can be detected through its gravitational effects with the nearby environment (galaxies), in particular, this means that dark matter does not interact directly with the standard matter (at least not with the ones contained in the standard particle model) [1]. While in the literature there are lots of particle-like models for dark matter, none of them seem to be able to properly describe dark matter completely at galactic scale or cosmological scales, then the only true physical information about dark matter is how it gravitationally interacts with normal matter (galaxies). Indeed, the gravitational lensing within a cluster of galaxies, reveals that the presence of concentrated dark matter in the inner zone of a cluster. Something that clearly cannot be predicted with ordinary matter within Newtonian theory because the mass of the galaxy is related with the circular rotation velocity $V$ through the Newton's law, namely the speed $V$ in a circular orbit at radius $r$ is related to the mass $M(<r)$ interior to that radius by the exact equation $M(<r)=r V^{2} / G$, being $G$ Newton's constant. One way to realize about the missing mass fact is by using

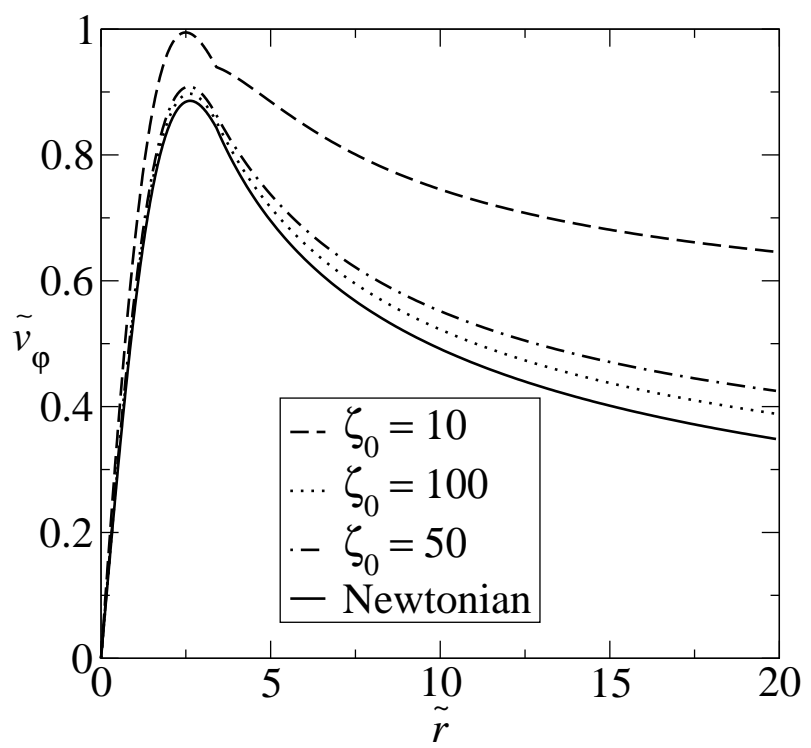

FIG. 6: Circular velocity rotation curves in terms of the dimensionless radial coordinate for different values of $\zeta_{0}$ two well-known procedures. To be more precise, applying the virial theorem to a cluster, one can determine the virial mass of the cluster and summing up all the mass of galaxies within the cluster one can estimate the baryonic mass. It turns out that the former one is 20 times bigger than the latter one, showing in this way the existence of additional (nonbaryonic) matter in the cluster [1].

So far we have focused on the basic features of selfgravitating systems of ideal gases in the $1 \mathrm{PN}$ approximation. Now, we devote our attention to the circular rotation curve due to its physical relevance in modern astrophysics at theoretical and observational levels, and in particular we examine the matching of two solutions at a critical radius, say $\widetilde{r}_{c}$. The idea behind such standard procedure is to reproduce the well-known observational features of the galaxy rotation curves, namely, a linear regime at small radii, passes through a cusp, and ends with a flatten shape at large radii. Let us now show how this shape can be obtained with the model at hand within the $1 \mathrm{PN}$ approximation. Fig. 2 shows that model describes well the inner zone corresponding to $\widetilde{r} \leq \widetilde{r}_{c}$ because the circular rotation curve grows linear with the radial distance, then it passes through small cusp and becomes ill-defined for $\widetilde{r}>\widetilde{r}_{c}+\epsilon$ with $\epsilon>0$, implying that the gravitational potentials become complex and therefore the solutions are unphysical. In order to solve this issue, we match these solutions with another two gravitational potentials, called $\widetilde{\phi}_{2}(\widetilde{r})$ and $\widetilde{\psi}_{2}(\widetilde{r})$. A somewhat natural question to ask is this one: which kind of physical equation should satisfy these new potentials? A quick answer would be that these ones must fulfill the Laplace equation, namely $\nabla^{2} \widetilde{\phi}_{2}(\widetilde{r})=0$ and $\nabla^{2} \widetilde{\psi}_{2}(\widetilde{r})=0$, and the potentials and theirs first derivatives must be glued at $\widetilde{r}_{c}$ in order to have a well-defined boundary problem. Let us analyze this proposal in detail. Taking into account that both potentials satisfy the Laplace equation, they can be recast as $\widetilde{\phi}_{2}(\tilde{r})=\alpha / \tilde{r}+\beta$ and $\widetilde{\psi}_{2}(\tilde{r})=\gamma / \tilde{r}+\delta$. After imposing the continuity of the potential and its derivative at $\widetilde{r}_{c}$, we arrived at $\alpha=-\widetilde{r}_{c}^{2} \widetilde{\phi}^{\prime}{ }_{1}\left(\widetilde{r}_{c}\right)$ along with $\beta=\widetilde{\phi}_{1}\left(\widetilde{r}_{c}\right)+\widetilde{r}_{c} \widetilde{\phi}_{1}^{\prime}\left(\widetilde{r}_{c}\right)$. The same thing holds for the other potential by replacing $\alpha \rightarrow \gamma, \beta \rightarrow \delta$ along with $\widetilde{\phi}_{1} \rightarrow \widetilde{\psi}_{1}$, where $\widetilde{\phi}_{1}$ and $\widetilde{\psi}_{1}$ are the gravitational potentials which satisfy the Poisson equations (36)-37) in the inner zone $\left(\widetilde{r} \leq \widetilde{r}_{c}\right)$. With this proposal at hand, we can extend physically our first solution beyond the critical radius, $\widetilde{r}_{c}$, however, we can prove that the aforesaid gravitational potentials do not reproduce the desired property of a flatten circular rotation curve in the outer zone. Replacing both potentials $\widetilde{\phi}_{2}(\widetilde{r})$ and $\widetilde{\psi}_{2}(\widetilde{r})$ into (45), one can check that the circular rotation curve goes to zero in the limit of large radii. Therefore, we need to introduce another type of potentials to do the job. In order to do so, we propose that $\widetilde{\phi}_{2}(\tilde{r})$ satisfies the Laplace's equation whereas $\widetilde{\psi}_{2}(\tilde{r})$ fulfills a Poisson's equation with the same boundary condition mentioned above. The lat- 
ter potential can be parametrized as

$$
\widetilde{\psi}_{2}(\tilde{r})=\gamma \frac{e^{-k \widetilde{r}}}{\widetilde{r}}+\delta \ln \widetilde{r} .
$$

Here $k$ is a parameter of the Yukawa term while $\gamma$ and $\delta$ are integration constants. The latter ones are obtained by solving the following linear system

$$
\left[\begin{array}{c}
\widetilde{\psi}_{2}\left(\tilde{r}_{c}\right) \\
\widetilde{\psi}_{2}^{\prime}\left(\tilde{r}_{c}\right)
\end{array}\right]=\left[\begin{array}{cc}
\frac{e^{-k \widetilde{r}_{c}}}{\widetilde{r}_{c}} & \ln \widetilde{r}_{c} \\
-k \frac{e^{-k \widetilde{r}_{c}}}{\widetilde{r}_{c}}-\frac{e^{-k \widetilde{r}_{c}}}{\widetilde{r}_{c}^{2}} & \frac{1}{\widetilde{r}_{c}}
\end{array}\right] \times\left[\begin{array}{l}
\gamma \\
\delta
\end{array}\right] .
$$

Using $\widetilde{\psi}_{2}\left(\tilde{r}_{c}\right)=\widetilde{\psi}_{1}\left(\tilde{r}_{c}\right)$ and $\widetilde{\psi}_{2}^{\prime}\left(\tilde{r}_{c}\right)=\widetilde{\psi}_{1}^{\prime}\left(\tilde{r}_{c}\right)$ we find the value of the integration constants numerically at $\tilde{r}_{c} \simeq 3.4001$, namely $\gamma \simeq-29.7253 \delta \simeq-2.6906$. It should be pointed that the aforesaid procedure is not dependent of the value taken by $\zeta_{0}$, however, the circular rotation curve is. As can be seen from Fig. 6, we find that the circular rotation curves flatten at very large radii for different values of $\zeta_{0}$. It is easy to understand how this proposal reproduces the flat shape by replacing the Coulomb potential $\widetilde{\phi}_{2}(\tilde{r})$ along with $\widetilde{\psi}_{2}(\tilde{r})$ into (45). The Coulomb and Yukawa terms fade away for large radii but the logarithm contribution introduces a constant in the circular velocity which dominates for large radii. In this way, we could include a Coulomb term in $\widetilde{\psi}_{2}(\tilde{r})$ instead of the Yukawa term and it will also lead us to the right flatten curve, however, the Yukawa term is better because it behaves smoother than the Coulomb potential for large radii. We end this section by mentioning some physical outcomes of our previous proposal. For $\widetilde{r} \geq \widetilde{r}_{c}$ the Newtonian density is given by $\widetilde{\rho}_{\text {mass }}=-\nabla^{2}{\widetilde{\phi_{2}}}_{2}=\alpha / \widetilde{r}^{2}$ which clearly goes to zero for large radius. In this zone, the post-Newtonian density and pressure are entangled provided we only know $\widetilde{\psi}_{2}$, that is, $\widetilde{\rho}_{P N}+\widetilde{p}_{P N}=-\nabla^{2} \widetilde{\psi}_{2}=-\gamma e^{-\widetilde{r}} / \widetilde{r}-\delta / \widetilde{r}^{2}$, where we have taken $k=1$ without loss of generality, then $\widetilde{\rho}_{P N}+\widetilde{p}_{P N}$ also vanishes in this limit. Notice that $\widetilde{\rho}_{P N}+\widetilde{p}_{P N}$ is always positive because $\gamma<0$ and $\delta<0$. All in all, we showed that rotation curves with a flatten profile can be obtained within the post-Newtonian approximation, starting from the Maxwell-Jüttner distribution function at $1 \mathrm{PN}$ and solving the corresponding gravitational potential at $1 \mathrm{PN}$ order. As a final remark, we should emphasize that in our approach the ergodic function used differs substantially from the polytrope profile explored in [8].

\section{CONCLUSIONS}

We have built an astrophysical model based on the Maxwell-Jüttner distribution function within the framework of general relativity which is described using postNewtonian formalism. In order to obtain this expression, we have integrated over the peculiar velocity 3D space, keeping only the relevant terms up to $1 \mathrm{PN}$ order. With this ergodic distribution at hand, we have obtained the general form of Newtonian density along with the postNewtonian density and pressure terms which enter in the energy-momentum tensor by using several Gaussian integrals (cf. Appendix B). This allowed us to demonstrate that our general expression coincides with the one reported by Weinberg [9], validating our procedure. As an example of how to apply this procedure, we considered the case of particle four-flow and calculated its temporal and spatial components.

From the energy density and pressure terms at $1 \mathrm{PN}$, we looked for static solution by analyzing the boundary value problem. We found that the energy density, pressure and gravitational potentials profiles in terms of dimensionless radial coordinate by solving the aforesaid equations numerically. In particular, we found the energy density vanishes for large radii but approaches to a constant value at the origin, further, such value becomes larger for smaller values of $\zeta_{0}$ [see Fig. 1]. Contrary to the case of polytropic ergodic distribution explored in [8], the energy density remains always positive at $1 \mathrm{PN}$ order. As part of the process of evaluating the circular velocity profiles, we obtained that post-Newtonian curves reach larger values in relation with the Newtonian case. In both cases, we have found that these curves exhibit a linear behavior near the center and then pass through a cusp [see Fig. 2]. Interestingly enough, we have found the behavior of the circular velocity for ideal gases differs from the one where the distribution function is characterized by a polytropic function of the energy [8], since in the latter case the values of the circular velocity in the $1 \mathrm{PN}$ approximation are smaller than the corresponding Newtonian approximation. In our case, the large values of the circular velocity in the $1 \mathrm{PN}$ approximation are due to the fact that the increase of the temperature of the gas, increases the thermal velocity of the particles of the gas $\sqrt{k T_{0} / m}$. We have examined the behavior of the dimensionless gravitational potential energy $\widetilde{U}$. So we have found that the Newtonian gravitational potential energy is always negative, while the post-Newtonian gravitational potential energies change their sign for large values of the radial distance from the configuration center [see Fig. 3. The temperature of the gas in the post-Newtonian term $\widetilde{\phi}^{2} / 2 \zeta_{0}$ determines the sign change of the gravitational potential energy. The gravitational potential energy exhibits the same behavior that the one associated with a polytropic distribution function with polytropic index $n=3$. In addition, we found a parametric profile of the equation of state $p(\rho)$ in terms of the dimensionless radial coordinate, see Fig. 5. At small radii, the ratio pressure-density becomes almost constant; increasing $\zeta_{0}$ the constant reaches larger values or equivalently the thermal energy of the ideal gas decreases considerably. The situation is reversed at large radii.

We have patched together two different kinds of gravitational potentials at a critical radius, called $\widetilde{r}_{c}$, in order to have a well-defined boundary problem, provided the potentials became complex beyond this radius. The 
physical motivation in dealing with this issue was to select gravitational potentials which reproduce a flatten behavior in the rotation curve at large radii, provided the linear and the cusp zones were already described by those potentials obtained by integrating numerically the energy density and pressure coming from the Maxwell-Jüttner distribution function at $1 \mathrm{PN}$. In doing so, we have shown that one of the potentials has a Coulomb form while the other one presents two kinds of terms, a Yukawa contribution plus a logarithmic term. Indeed, the term responsible for the constant circular velocity at large radii turned out to be the logarithmic term. Regarding the changes introduced by these solutions, we have found that the Coulomb potential led to an inverse square power law for the Newtonian density, as one could expect. However, we could not disentangle the post-Newtonian density from the post-Newtonian pressure, thus we found $\widetilde{\rho}_{P N}+\widetilde{p}_{P N}=-\gamma e^{-\widetilde{r}} / \widetilde{r}-\delta / \widetilde{r}^{2}$ which leads to a positive quantity, vanishing for large radii only.

\section{Acknowledgments}

G. M. K. and M. G. R. are supported by Conselho Nacional de Desenvolvimento Científico e Tecnológico (CNPq) and K. W. by Coordenação de Aperfeiçoamento de Pessoal de Nível Superior (CAPES). G. M. K. thanks J. F. Pedraza for suggestions.

\section{Appendix A: SEVERAL QUANTITIES AT 1PN}

Up to the 1PN fourth order the components of the metric tensor are given by [9]

$$
\begin{aligned}
& g_{00}=-1+\stackrel{2}{g}_{00}+\stackrel{4}{g}_{00}+\ldots \\
& g_{i j}=\delta_{i j}+\stackrel{2}{g}_{i j}+\stackrel{4}{g}_{i j}+\ldots \\
& g_{0 i}=\stackrel{3}{g}_{0 i}+\stackrel{5}{g}_{0 i}+\ldots \\
& \stackrel{2}{g}_{00}=-2 \frac{\phi}{c^{2}}, \quad \stackrel{4}{g}_{00}=-\frac{2}{c^{4}}\left(\phi^{2}+\psi\right), \\
& \stackrel{2}{g}_{i j}=-2 \delta_{i j} \frac{\phi}{c^{2}}, \quad \stackrel{3}{g}_{0 i}=\frac{\xi_{i}}{c^{3}} \\
& \sqrt{-g}=1-\frac{2 \phi}{c^{2}}-\frac{\phi^{2}-\psi}{c^{4}} .
\end{aligned}
$$

Here $\phi$ is the Newtonian gravitational potential, and $\psi$ and $\xi_{i}$ are gravitational potentials in the $1 \mathrm{PN}$ approximation. These gravitational potentials are connected with the energy-momentum tensor by Einstein's field equations.

\section{Appendix B: GAUSSIAN INTEGRALS}

Let us summarize the most interesting cases that we have employed within this article. Just to make things more familiar, let us consider integration over a $3 \mathrm{D}$ space associated to the peculiar velocity space defined as $W *=$ $\left\{\left(w^{*}, \theta, \phi\right): 0 \leq w^{*} \leq \infty, 0 \leq \theta \leq 2 \pi, 0 \leq \phi \leq \pi\right\}$, where $w^{*}$ is a dimensionless peculiar velocity and the volume element is given by $d^{3} W^{*}=w^{* 2} d w^{*} d \Omega_{2}$ with $d \Omega_{2}$ the 2-dimensional element of solid angle. Let us consider the Gaussian distribution $F\left(w^{*}\right)=e^{-w^{* 2} / 2}$ so the following expressions can be obtained:

$$
\begin{aligned}
& I_{0}=\int e^{-\frac{w^{* 2}}{2}} d^{3} W^{*}=4 \pi \sqrt{\frac{\pi}{2}}, \\
& I_{2}=\int w^{* 2} e^{-\frac{w^{* 2}}{2}} d^{3} W^{*}=12 \pi \sqrt{\frac{\pi}{2},} \\
& I_{4}=\int w^{* 4} e^{-\frac{w^{* 2}}{2}} d^{3} W^{*}=60 \pi \sqrt{\frac{\pi}{2}}, \\
& I_{6}=\int w^{* 6} e^{-\frac{w^{* 2}}{2}} d^{3} W^{*}=420 \pi \sqrt{\frac{\pi}{2}} .
\end{aligned}
$$

Using (58), (59), and (60) we can prove that the following expressions hold (see e.g. [20]):

$$
\begin{aligned}
& \int F\left(w^{*}\right) w_{i}^{*} w_{j}^{*} d^{3} W^{*}=\frac{I_{2}}{3} \delta_{i j}, \\
& \int F\left(w^{*}\right) w_{i}^{*} w_{j}^{*} w_{k}^{*} w_{l}^{*} d^{3} W^{*} \\
& =\frac{I_{4}}{15}\left[\delta_{i j} \delta_{k l}+\delta_{i k} \delta_{j l}+\delta_{i l} \delta_{j k}\right] \\
& \int F\left(w^{*}\right) w_{i}^{*} w_{j}^{*} w_{k}^{*} w_{l}^{*} w_{m}^{*} w_{n}^{*} d^{3} W^{*} \\
& =\frac{I_{6}}{105}\left[\delta_{i j} \delta_{k l} \delta_{m n}+\delta_{i j} \delta_{k m} \delta_{l n}+\delta_{i j} \delta_{k n} \delta_{l m}\right. \\
& +\delta_{i k} \delta_{j l} \delta_{m n}+\delta_{i k} \delta_{j m} \delta_{l n}+\delta_{i k} \delta_{j n} \delta_{l m}+\delta_{i l} \delta_{j k} \delta_{m n} \\
& +\delta_{i l} \delta_{j m} \delta_{k n}+\delta_{i l} \delta_{j n} \delta_{k m}+\delta_{i m} \delta_{j k} \delta_{l n}+\delta_{i m} \delta_{j l} \delta_{k n} \\
& \left.+\delta_{i m} \delta_{j n} \delta_{k l}+\delta_{i n} \delta_{j k} \delta_{l m}+\delta_{i n} \delta_{j l} \delta_{k m}+\delta_{i n} \delta_{j m} \delta_{k l}\right] .
\end{aligned}
$$

[1] L. S. Sparke and J. S. Gallagher III, Galaxies in the Universe. Cambridge University Press (2007).

[2] A. Liddle, An Introduction to Modern Cosmology. John Wiley and Sons Inc. (2003); Hans-Volker Klapdor and Richard Arnowitt, Dark matter in Astro-and Particle Physics. Springer (2004).

[3] J. Jalocha, L. Bratek, M. Kutschera, P. Skindzier, Global disc models for galaxies NGC 1365, 6946, 7793 and UGC 6446, MNRAS 406, 2805 (2010); J. Jalocha, L. Bratek, M. Kutschera, P. Skindzier, Vertical gradients of azimuthal velocity in a global thin-disc model of spiral galaxies NGC 2403, NGC 4559, NGC 4302 and NGC 5775, MNRAS 412, 331 (2011); J. Jalocha, S. Sikora, L. Bratek, M. Kutschera, Constraining the vertical struc- 
ture of the Milky Way rotation by microlensing in a finitewidth global disk model, AA 566, A87 (2014).

[4] Persic, M., Salucci, P., Stel, F. The universal rotation curve of spiral galaxies - I. The dark matter connection. MNRAS Vol. 281, Issue 1, pp. 27-47 (1996).

[5] C. Cercignani and G. M. Kremer, The relativistic Boltzmann equation: theory and applications. Birkhäuser, Basel (2002).

[6] W. Zimdahl and G. M. Kremer, Temperature oscillations of a gas in circular geodesic motion in the Schwarzschild field, Phys. Rev. D 91, 024003 (2015).

[7] Marcel Zemp, Collisionless and collisional dynamics in astrophysics, ETH Zurich, Master thesis (2006).

[8] C. A. Agón, J. F. Pedraza and J. Ramos-Caro, Kinetic theory of collisionless self-gravitating gases: postNewtonian polytropes, Phys. Rev. D 83, 123007 (2011).

[9] S. Weinberg, Gravitation and cosmology, principles and applications of the general theory of relativity. John Wiley \& Sons, New York (1972).

[10] H. Balasin and D. Grumiller, Non-Newtonian behavior in weak field general relativity for extended rotating sources, Int. J. Mod. Phys. D, 17, 475 (2008).

[11] J. Ramos-Caro, C. A. Agón and J. F. Pedraza, Kinetic theory of collisionless self-gravitating gases. II. Relativis- tic corrections in galatic dynamics, Phys.. Rev. D 86, 043008 (2012).

[12] P.H. Nguyen and J. F. Pedraza, Anisotropic models for globular cluster, Galatic bulges, and dark halos, Phys. Rev. D 88, 064020 (2013).

[13] N. A. Chernikov, The relativistic gas in the gravitational field, Acta Phys. Pol., 23, 629 (1963).

[14] J. Ehlers (Ed.), Relativity theory and Astrophysics 1Relativity and cosmology, Vol. 8, American mathematical society, University of Texas, 1967.

[15] L. Bel, Kinetic theory of cosmology, Ap. J.155, 83 (1969).

[16] W. Israel, The relativistic Boltzmann equation, in General relativity, ed. L. O'Raifeartaigh. Clarendon Press, Oxford (1972).

[17] R. C. Tolman, On the weight of heat and thermal equilibrium in general relativity, Phys. Rev. 35, 904 (1930).

[18] R. C. Tolman and P. Ehrenfest, Temperature equilibrium in a static gravitational field, Phys. Rev. 36, 1791 (1930).

[19] O. Klein, On the thermodynamical equilibrium of fluids in gravitational fields, Rev. Mod. Phys. 21, 531 (1949).

[20] G. M. Kremer, An introduction to the Boltzmann equation and transport processes in gases. Springer, Berlin (2010). 